\title{
DIAGNÓSTICOS DE ENFERMAGEM DE PACIENTES EM UNIDADES DE TERAPIA INTENSIVA*
}

\author{
Dolores Pasini** \\ Iracema Alvim** \\ Luiza Kanda** \\ Rita do Socorro Pereira Mendes** \\ Diná de Almeida Lopes Monteiro da Cruz***
}

PASINI, D. et al Diagnósticos de enfermagem de pacientes internados em unidades de terapia intensiva (UTI). Rev. Esc. Enf. USP, v.30 ,n.3, p.501-18, dez. 1996.

Com a finalidade de caracterizar as necessidades de cuidados de doentes de unidades de terapia intensiva foram realizadas entrevistas e exames físicos junto a 32 pacientes e formulados os seus diagnósticos de enfermagem. Foi utilizado o referencial dos Padrões Funcionais de Saúde para a coleta dos dados e para a caracterização das áreas em que as alterações são predominantes.

UNITERMOS: Diagnóstico de enfermagem. Unidade de terapia intensiva. Padrões funcionais de saúde.

\section{INTRODUÇÃO}

Em 1973 foi realizada a $1^{\mathrm{a}}$ Conferência sobre Diagnósticos de Enfermagem na América do Norte que teve como objetivos identificar e denominar as situações ou os fenômenos clínicos nos quais os enfermeiros poderiam intervir (GEBBIE; LAVIN, 1995). Nessa conferência foram sugeridos diagnósticos pela denominação das situações clínicas dos pacientes que os enfermeiros encontravam na prática de enfermagem. Foram aceitos como diagnosticos de enfermagem as situações que a maioria dos participantes consideram suficientemente claras para serem testadas clinicamente. Nessa primeira conferência foram aceitos cerca de 30 diagnósticos.

Em 1982 houve a criação da North American Nursing Diagnosis Association (NANDA) com a finalidade de reunir esforços para denominar, classificar e

\footnotetext{
* Trabalho apresentado ao X Curso de Especializaçáo em Enfermagem em Cuidados Intensivos

* * Enfermeira. Aluna do X Curso de Especializaçáo em Enfermagem em Cuidados Intensivos

*** Enfermeira. Professora Doutora do Departamento de Enfermagem Médico-Cirúrgica da Escola de Enfermagem da Universidade de São Paulo
} 
desenvolver as questões referentes ao diagnóstico de enfermagem. Os diagnásticos de enfermagem, que vêm sendo desenvolvidos pela North American Nursing Association (NANDA), são uma forma de discriminar os fenômenos de interesse para a enfermagem, uma vez que designam e descrevem as respostas dos pacientes aos problemas de saúde ou aos processos vitais. A idéia de diagnóstico de enfermagem cobre a lacuna existente entre a coleta de informações e o planejamento de cuidados, pela inserção do julgamento clínico e inferência feitos pelo profissional sobre os dados coletados. Essa autora afirma que o diagnóstico de enfermagem descreve problemas de saúde reais ou potenciais que os enfermeiros, em virtude de sua formação e experiência, são capazes e legalmente habilitados para tratar (GORDON, 1987).

Para MUNDINGER (1978) o diagnóstico de enfermagem é função específica do enfermeiro, mas o ato de diagnosticar segue o mesmo processo para todos aqueles que o fazem, isto é, é o processo de inferir a natureza e a causa de um estado indesejado de coisas. Afirma ainda, que além de necessitar de conhecimento para fazê-lo também deve possuir habilidade para resolver a situação problemática ou indes ejável.

Bienalmente têm ocorrido conferências para rever e classificar diagnósticos de enfermagem. Os estudos continuam para que se validem esses diagn6sticos e se identifiquem outros a partir da prática de enfermagem (GORDON et al, 1980). Em 1990 estavam aceitos 99 diagnósticos de enfermagem e a seguinte definição foi proposta: diagnóstico de enfermagem é o "julgamento clínico das respostas do indivíduo, da família ou da comunidade aos processos vitais ou aos problemas de saúde reais ou potenciais. Os diagnósticos de enfermagem fornecem a base para a seleção de intervenções para atingir resultados pelos quais o enfermeiro é responsável" (MILLS 1991).

Os diagnósticos de enfermagem foram agrupados em 9 categorias denominadas de padrões de respostas humanas que são: trocar, comunicar, mover, relacionar, valorizar, perceber, sentir, conhecer e escolher. Cada diagnóstico possui definição, listagem de suas características definidoras e de fatores a ele relacionados (NORTH AMERICAN NURSING DIAGNOSIS ASSOCIATION, 1994).

A proposição dos padrões de respostas humanas só surgiu em 1986 e são ainda pouco familiares para os profissionais. Em 1982 GORDON propôs uma organização dos diagnósticos de enfermagem aceitos que continua bastante difundida. Buscando delinear áreas básicas de coleta de informações significativas para a enfermeira e aplicáveis a todos os pacientes GORDON (1982) propôs uma série de onze categorias nominais, representando áreas básicas de saúde que auxiliam na coleta dos dados para a elaboração de diagnósticos de enfermagem. Essas áreas representam idéias tradicionais e contemporâneas da prática de enfermagem. Foram denominadas de padrões funcionais de saúde que podem ser descritos a partir de dados clínicos obtidos pela história e exame físico realizado pelo enfermeiro. Esses padrões funcionais de saúde são: padrão de percepção e controle de saúde, padrão nutricional-metab6lico, padrão de eliminações, padrão cognitivo-perceptivo, padrão de autopercepção e autoconceito, padrão de 
desempenho de papel e relacionamento, padrão sexual-reprodutivo, padrão de resposta e tolerância ao estresse, padrão de crença e valor, padrão de atividade e exercício e padrão de sono e repouso. Com essa proposta de abordagem de coleta de informações e considerando que o seu propósito é identificar as necessidades de cuidados dos doentes GORDON (1982) classifica os diagnósticos de enfermagem aceitos pela NANDA na mesma estrutura que norteia a coleta dos dados.

O uso da classificaşão dos diagnósticos de enfermagem na mesma estrutura da coleta dos dados favorece o processo diagnóstico, porque ao se analisar e sintetizar os dados referentes a um determinado padrão pode-se considerar todas as possibilidades diagnósticas desse mesmo padrão. No entanto, isso não significa que os dados coletados num padrão não alimentem o processo de raciocínio sobre outros padrões.

Cada diagnóstico aceito pela NANDA tem uma definição que busca explicitar a idéia ou o significado do nome do diagnóstico. Isso favorece o entendimento consistente dos termos e fornece base para revisão e refinamento de conceitos. Além da definição, cada diagnóstico de enfermagem é apresentado com uma listagem de características definidoras e ou tra de fatores relacionados. As características definidoras são as evidências que o enfermeiro identifica no paciente a partir do levantamento de dados e que pelo seu julgamento interpreta e agrupa; elas são fundamentais para a afirmação diagnostica. Alguns diagnósticos têm características definidoras críticas, isto é sem presença delas não se pode afirmar o diagnóstico em questão. Os fatores relacionados são elementos que contribuem para a ocorrência do diagnóstico. Devem ser investigados pois auxiliam na determinação das intervenções. Os diagnósticos podem ser atuais ou potenciais. Os potenciais, como se referem a situações de risco do paciente e não a respostas já apresentadas, não têm características definidoras ou fatores relacionados, mas têm fatores de risco que, tanto são a base para afirmá-los quanto os fatores que contribuem para o alto risco (CRUZ, 1994).

No Brasil, o conhecimento dos profissionais da área sobre diagnóstico de enfermagem é emergente pelo fato das publicações nacionais sobre 0 assunto serem do início desta década e ainda escassas (CRUZ, 1989; MARIA, 1990; FARIAS et al, 1990; CRUZ, 1993; PIMENTA, CRUZ, 1994).

Poucos trabalhos descrevem os diagnósticos de enfermagem de pacientes em unidades de terapia intensiva (GORDON, 1994; GREENLEE, 1991). Segundo WIESEKE et al (1994) uma revisão de periódicos de enfermagem em cuidados críticos revelou pouca discussão sobre o diagnóstico de enfermagem como guia para o julgamento na assistência ao paciente crítico. No Brasil, conhecemos apenas o trabalho de BUB et al (1995) que se refere a diagnósticos de enfermagem junto à pacientes de unidades de terapia intensiva.

A descrição dos diagnosticos de enfermagem de doentes de unidades de terapia intensiva fornece um perfil das necessidades de cuidados desses doentes sobre o qual é possível organizar o conhecimento necessário na área, além de 
permitir comparações entre populaçōes específicas de doentes. Assim visando descrever parte da demanda de cuidados de enfermagem em unidade de terapia intensiva o presente estudo teve os objetivos:

1.Identificar a frequência dos diagnósticos de enfermagem de pacientes conscientes em unidades de terapia intensiva.

2.Identificar a frequência de alterações dos padrões funcionais de saúde de pacientes em unidades de terapia intensiva.

\section{MATERIAL E MÉTODO}

Os dados foram coletados junto a pacientes em unidades de terapia intensiva gerais, no ano de 1995 , em instituições públicas e privadas na cidade de São Paulo. A população foi composta por conveniência de acordo com os requisitos: ser adulto maior de 20 anos, ter condições de verbalização, estar internado há no máximo dez dias na U'TI, ter disponibilidade e concordar em participar do estudo.

A coleta dos dados foi feita por entrevista e exame físico utilizando-se instrumento elaborado especificamente para esse fim que constou de questões abertas e fechadas abordando os 11 Padrões Funcionais de Saúde e de um guia para exame físico conforme proposto por GORDON (1994) (Anexo I).

A análise e síntese dos dados para formular os diagnósticos de enfermagem foram feitas pelo próprio pesquisador que coletou os dados. Foram incluídos as diagnósticos para os quais houve consenso entre os autores deste estudo.

\section{RESULTADOS E DISCUSSÃO}

Os resultados encontrados referem-se a uma população de 32 pacientes, com idade média de 52,5 anos sendo $65,6 \%$ do sexo masculino. A escolaridade da população foi predominantemente curso primário (53\%). Os diagnósticos médicos principais desses doentes se referiam aos sistemas: cardiovascular $(33,7 \%)$, digestivo $(28,3 \%)$, endócrino $(12,5 \%)$ e outros $(15,5 \%)$.

Foram formuladas 31 categorias diferentes de diagnósticas de enfermagem. O total de diagnósticos de enfermagem foi 256, com média de 8 diagnósticos de enfermagem por paciente. A variação de diagnósticos por paciente foi de 4 à 12 . Esses resultados são semelhantes aos de outros estudos de perfil de diagnósticos de enfermagem em outras populações de pacientes em outras unidades de internação. Talvez essas semelhanças sejam decorrentes dos critérios adotados para o presente estudo. Vários pacientes tinham diagnóstico médico de Infarto Agudo do Miocárdio e outros com poucos dias de internação na UTI o que pode ter aproximado esses resultados dos que têm sido encontrados em pacientes em unidades de internação geral (CRUZ, 1989) ou em unidades de pacientes externos (PIMENTA; CRUZ, 1994). 
Houve diagnósticos reais e potenciais. Os diagnósticos reais referem-se àquelas respostas já presentes nos pacientes e os diagnósticos potenciais referemse a situações para as quais os pacientes apresentam alto risco para a resposta em questão.

O QUADRO 1 mostra as categorias dos diagnósticos de enfermagem da população e as respectivas frequências.

QUADRO 1 - Freqüência das categorias dos diagnósticos de enfermagem formulados para 32 pacientes internados em unidades de terapia intensiva. São Paulo, 1995.

\begin{tabular}{l|l|l}
\multicolumn{1}{c|}{ Categorias de Diagnósticos } & \multicolumn{2}{c}{ Pacientes } \\
\hline 1. Alto risco para infecção & 31 & 96,8 \\
\hline 2. Integridade da pele prejudicada & 31 & 96,8 \\
\hline 3. Mobilidade física prejudicada & 26 & 81,2 \\
\hline 4. Distúrbio no padrão do sono & 24 & 75 \\
\hline 5. Dor & 20 & 62,5 \\
\hline 6. Intolerância a atividade & 14 & 43,5 \\
\hline 7. Déficit para auto-cuidado (banho,vestir, eliminações) & 14 & 43,5 \\
\hline 8. Déficit para conhecimento (da doença) & 13 & 40,6 \\
\hline 9. Obstipação intestinal (constipação) & 11 & 34,3 \\
\hline 10. Ansiedade & 9 & 24,1 \\
\hline 11. Alteração na mucosa oral & 7 & 21,8 \\
\hline 12. Alto risco para aspiração & 6 & 18,7 \\
\hline 13. Alto risco para déficit de volume líquido & 6 & 18,7 \\
\hline 14. Diarréia & 5 & 15,6 \\
\hline 15. Alteração na nutrição para maior que o corpo necessita & 4 & 12,5 \\
\hline 16. Alteração senso perceptivo (audição) & 4 & 9,3 \\
\hline 17. Excesso de volume de líquido & 3 & 12,5 \\
\hline 18. Medo & 4 & \multicolumn{1}{c}{ N } \\
\hline
\end{tabular}




\begin{tabular}{l|l|l}
\multicolumn{1}{c|}{ Categorias de Diagnósticos } & \multicolumn{2}{c}{ Pacientes } \\
\hline 19. Deglutição prejudicada & 3 & 9,3 \\
\hline 20. Padrão respiratório ineficaz & 3 & 9,3 \\
\hline 21. Alteração senso perceptivo (visual) & 3 & 9,3 \\
\hline 22. Comunicação verbal dificultada & 2 & 6,2 \\
\hline 23. Impotência & 2 & 6,2 \\
\hline 24. Potencial para trauma & 2 & 6,2 \\
\hline 25. Hiperternia & 2 & 6,2 \\
\hline 26. Alteração na nutrição para menos que o corpo necessita & 2 & 6,2 \\
\hline 27. Alteração no padrão da sexualidade & 1 & 3,1 \\
\hline 28. Eliminação traqueo-brônquica inelicaz & 1 & 3,1 \\
\hline 29. Alteração na manutenção da saúde & 1 & 3,1 \\
\hline 30. Alteração na perfusão tecidual & 1 & 3,1 \\
\hline 31. Alteração dá eliminação urinária & 1 & 3,1 \\
\hline
\end{tabular}

Observa-se que $96,8 \%$ dos pacientes do estudo apresentou alto risco para infecção, integridade da pele prejudicada e que os outros diagnosticos mais frequentes foram: mobilidade física prejudicada $(81,2 \%)$, distúrbio no padrão do sono $(75,0 \%)$ e dor $(62,5 \%)$. A intolerância a atividade e o déficit para autocuidado (banho, vestir, eliminações) foram identificados em 43,5\% dos pacientes e o déficit de conhecimento (da doença) em 40,6\%. Os diagnósticos referentes ao autocuidado foi nesse estudo agrupado porque os pacientes apresentavam o deficit simultaneamete nas três áreas (banhar-se, vestir-se e cuidar-se nas eliminações) embora eles sejam diagnósticos separados por exigirem diferentes intervenções. Esse quadro mostra ainda que 14 categorias diagnosticas (45,2\% do total) foram formuladas para $3(10 \%)$ pacientes ou menos, indicando que cada paciente tem uma conformação específica, individual de necessidades de cuidado.

A formulação de diagnóstico de enfermagem fundamenta-se na identificação de sinais e sintomas que os caracterizam. SHOEMAKER (1985) afirma que há necessidade de identificar os sinais e sintomas que devem, necessariamente, estar presentes para que um determinado diagnóstico seja confirmado. Esses sinais e sintomas seriam os indicadores críticos dos diagnósticos. Quando os indicadores críticos forem determinados para cada diagnóstico, o processo diagnóstico em si será facilitado (MORRIS, 1985) e o erro reduzido (VINCEN'T, 1985). 
Nem todos os diagnósticos têm a especificação dos indicadores críticos e por essa razão há várias publicações de estudos realizados com esse objetivo (CREASON et al 1985; HOSKINS et al, 21986; McDONALD, 1985; MUNNS, 1985; VOITH; SMITH, 1985).

Apresentamos a seguir as características definidoras e fatores relacionados dos diagnósticos apresentados por mais de $50 \%$ da população.

QUADRO 2 - Freqüência dos fatores de risco que fundamentaram o diagnóstico de alto risco para infecção em pacientes em UTI. São Paulo, 1995.

\begin{tabular}{|c|c|c|c|c|}
\hline Diagnóstico & $\mathbf{N}^{\circ}$ & Fatores de Risco & $\mathbf{N}^{0}$ & $\%$ \\
\hline $\begin{array}{l}\text { - Alto risco para } \\
\text { infecção }\end{array}$ & 31 & $\begin{array}{l}\text { - Solução de continuidade da pele } \\
\text { - Restrição no leito } \\
\text { - Diminuição de murmúrios vesiculares em bases } \\
\text { pulmonares } \\
\text { - Prejuízo do sistema imunológico } \\
\text { - Lesão da mucosa oral } \\
\text { - Síbilos à ausculta pulmonar } \\
\text { - Estertores à ausculta pulmonar } \\
\text { - Prejuízo na perfusão tecidual } \\
\text { - Eliminação traqueo-brônquica ineficaz }\end{array}$ & $\begin{array}{l}30 \\
09 \\
07 \\
04 \\
02 \\
02 \\
02 \\
01 \\
01\end{array}$ & $\begin{array}{c}96,7 \\
29,0 \\
22,5 \\
12,9 \\
6,4 \\
6,4 \\
6,4 \\
3,2 \\
3,2\end{array}$ \\
\hline
\end{tabular}

O diagnóstico de alto risco de infecção é definido como o estado em que o indivíduo está com risco aumentado para ser invadido por microorganismos patogênicos (FARIAS 1990). Dos 31 pacientes, 30 (96,7\%) tinham solução de continuidade da pele por procedimentos invasivos, feridas cirúrgicas, escarificações por compressão ou assaduras. A restrição no leito; diminuição do murmúrio vesicular em bases pulmonares e prejuízo do sistema imunológico também foram identificados como fatores de risco para o diagnóstico. Era esperado que esse diagnóstico fosse frequente nos pacientes de UTI porque eles geralmente necessitam de procedimentos diagnósticos ou terapêuticos invasivos. O prejuízo da mobilidade do paciente também é fator de risco para este diagnóstico porque favore o desenvolvimento de congestão pulmonar, propiciando infecções e atelectasias. Em pesquisa realizada com 678 enfermeiros americanos de unidades de terapia intensiva, observou-se que este diagn6stico foi indicado por $92 \%$ da população como uma resposta frequentemente presente na prática diária em unidade de terapia intensiva (GORDON, 1994). GALVÃO; CRUZ (1995) Também identificaram esse diagnóstico como um dos mais frequentes em doentes internados em diversas unidades de hospital especializado. 
QUADRO 3 - Freqüência das características que fundamentaram o diagnóstico de integridade da pele prejudicada em pacientes de UTI. São Paulo, 1995.

\begin{tabular}{l|c|l|c|c}
\hline \multicolumn{1}{c|}{ Diagnóstico } & $\mathbf{N}^{\mathbf{0}}$ & \multicolumn{1}{|c|}{ Características } & $\mathbf{N}^{\circ}$ & $\%$ \\
\hline $\begin{array}{l}\text { Integridade da pele } \\
\text { prejudicada }\end{array}$ & 31 & $\begin{array}{l}\text { - Solução de continuidade da pele (jelco, incisão, } \\
\text { cirurgia, assadura) } \\
\text { - Hematoma de maxilar } \\
\text { - Urticária }\end{array}$ & 31 & 100 \\
& & 01 & 3,2 \\
\hline
\end{tabular}

Observa-se no QUADRO 3 que o diagnóstico de integridade da pele prejudicada foi formulado para 31 pacientes. Esse diagnóstico é definido como o estado em que a pele do indivíduo está prejudicada (FARIAS 1990). Os 31 (100\%) pacientes com esse diagnóstico apresentaram solução de continuidade da pele por procedimentos invasivos e lesões cutâneas.

Todos os pacientes com alto risco para infeç̧ão tiveram o diagnóstico de prejuízo da integridade da pele. Se considerarmos que as intervenções para os dois diagnósticos podem ser as mesmas caso as características definidoras do último sejam os fatores de risco do primeiro, questionamos se é legítimo os indicarmos como duas respostas separadas.

QUADRO 4 - Freqüência dos fatores relacionados do diagnóstico de integridade da pele prejudicada em pacientes de UTI. São Paulo, 1995.

\begin{tabular}{l|c|l|c|c}
\hline \multicolumn{1}{c|}{ Diagnóstico } & $\mathbf{N}^{\mathbf{0}}$ & \multicolumn{1}{|c|}{ Características } & $\mathbf{N}^{\mathbf{0}}$ & $\%$ \\
\hline $\begin{array}{l}\text { Integridade da pele } \\
\text { prejudicada }\end{array}$ & 31 & - Procedimentos invasivos & 31 & 100 \\
& & - Lesões cutâneas (assaduras e escaras) & 02 & 6,4 \\
\hline
\end{tabular}

O fator relacionado para integridade da pele prejudicada foi para a totalidade. dos pacientes com esse diagnóstico proveniente de procedimentos invasivos. As lesões cutâneas não decorrentes de procedimentos invasivos também foram fatores relacionados que se associaram aos primeiros. Uma vez que nas unidades de terapia intensiva são rotineiramente utilizados procedimentos invasivos para monitorizar o paciente e tratar diferentes desequilíbrios era de se esperar uma prevalência acentuada desse fator, perfazendo $100 \%$. As lesões cutâneas encontradas na população estudada, estavam ligadas a: imobilizações prolongadas no leito levando a formações de úlceras de pressão. As assaduras foram decorrentes de episodios diarréicos ou ainda ligadas ao excesso de peso do paciente e às fixações de drenos e curativos perfazendo um total de $6,4 \%$. Tornase importante frisarmos que essa frequência pode estar diminuída devido aos critérios utilizados para selecionar a população de estudo. 
QUADRO 5 - Freqüência das características que fundamentaram o diagnóstico de mobilidade física prejudicada em pacientes de U.T.I. - São Paulo, 1995.

\begin{tabular}{c|c|l|c|c}
\hline Diagnóstico & $\mathbf{N}^{0}$ & \multicolumn{1}{|c|}{ Características } & $\mathbf{N}^{\mathbf{0}}$ & $\%$ \\
\hline Mobilidade física & 26 & - Não consegue se movimentar independentemente & & \\
& & no leito & 17 & 65,3 \\
& & - Restrição à movimentação & 10 & 38,4 \\
& & - Medo de movimentar-se no leito & 09 & 34,6 \\
& & - Sonolento & 01 & 3,8 \\
& & - Fraqueza & 01 & 3,8 \\
\hline
\end{tabular}

O diagnóstico de mobilidade física prejudicada é definido como o estado em que o indivíduo experimenta uma limitação na habilidade para movimentos físicos independentes (FARIAS, 1990). Observa-se no QUADRO 5 que dos 26 pacientes $17(65,3 \%)$ não conseguiam se movimentar independentemente no leito, $10(38,4 \%)$ tinham restrição d̀ movimentação por dor e $9(34,6 \%)$ sentiam medo de movimentar-se no leito. Esse diagnóstico foi o terceiro em maior frequência nessa população. Apesar de os pacientes serem conscientes, e muitas vezes não terem uma limitação aos movimentos impostas pelas capacidades físicas, o próprio tratamento em terapia intensiva impõe limitações à habilidade do paciente movimentar-se independentemente como por exemplo o uso de monitor de eletrocardiograma, venóclise, sondagens. De qualquer forma merece ainda discussão se se trata da mesma resposta quando o prejuízo na mobilidade física advém de limitação inerente ao paciente. Nesse sentido, destacamos que consideramos diferentemente essa questão ao formularmos os diagnósticos de déficit de autocuidado. Nesse caso, com base nas definições que envolvem explicitamente a incapacidade do paciente em desempenhar as atividades a que o diagnóstico se refere, consideramos que quando havia limitações impostas pelo tratamento os diagnósticos de déficit de autocuidado não existiam. Mesmo assim é discutível essa opção. Os doentes com intolerância à atividade manifesta por dor anginosa, arritmias e que estavam nos primeiros dias do infarto agudo do miocárdio, quando se recomenda banho no leito, por exemplo, não tiveram o diagnóstico de déficit de autocuidado para banho formulado. Nessa situação podese discutir tanto as definições dos diagnósticos, a propriedade destes resultados, como o fato de determinadas intervenções não serem necessariamente vinculadas a um diagnóstico específico.

QUADRO 6-Freqüência dos fatores relacionados do diagnóstico de mobilidade física em pacientes internados em UTI. São Paulo, 1995.

\begin{tabular}{l|c|l|c|c}
\hline \multicolumn{1}{c|}{ Diagnóstico } & $\mathbf{N}^{\bullet}$ & \multicolumn{1}{|c|}{ Fatores relacionados } & $\mathbf{N}^{\circ}$ & $\%$ \\
\hline Mobilidade física & 26 & - Imposiçāo do tratamento & 15 & 57,6 \\
prejudicada & & - Dor & 10 & 38,4 \\
& & - Intolerância a atividade & 04 & 15,3 \\
& & - Falta de conhecimento & 02 & 7,6 \\
& & - Dano neurológico & 01 & 3,8 \\
\hline
\end{tabular}


Observa-se no QUADRO 6 que nessa população o diagnóstico de mobilidade física prejudicada estava predominantemente relacionado à imposição do tratamento $(57,6 \%$ dos pacientes com esse diagnóstico). Foi referido por alguns pacientes que eles se sentiam aptos para atividades físicas que gostariam de realizar mas que fazia parte das recomendações do tratamento a restrição ao leito. Dessa forma, para esses pacientes a imposição do tratamento foi o fator relacionado do diagnóstico em questão. A dor, embora presente em 20 (100\%) doentes, foi fator relacionado da mobilidade física prejudicada em dez casos. Como já foi observado por PIMEN'TA; (KRUZ (1994) a dor parece ser diagnóstico nuclear em torno do qual ocorrem outras respostas como a mobilidade física prejudicada. Depreende-se também do QUAIRRO 6 que a mobilidade física prejudicada teve mais de um fator relacionado para alguns pacientes. É comum na prática clínica observarmos diferentes fatores do ambiente, do próprio paciente ou da interação ambos contribuindo para a ocorrência ou manutenção de uma resposta que necessita de cuidados de enfermagem.

Embora para 2 pacientes tenhamos indicado o déficit de conhecimento como fator contribuinte para a mobilidade física prejudicada é discutível a validade de utilizarmos um diagnóstico de enfermagem como fator relacionado de outro. Apesar disso, entendemos que se as duas respostas ocorrem como neste caso - mobilidade física prejudicada e déficit de conhecimento - éválido indicarmos as duas, no mínimo para que conheçamos adequadamente as reações do pacientes às intervenções propostas. No caso desses dois pacientes, eles, por desconhecimento, limitavam a própria mobilidade física.

QUADRO 7 - Freqüência das características que fundamentaram o diagnóstico de distúrbio no padrão do sono de pacientes internados em UTI. São Paulo, 1995.

\begin{tabular}{l|c|l|c|c}
\hline \multicolumn{1}{c|}{ Diagnóstico } & $\mathbf{N}^{\mathbf{0}}$ & \multicolumn{1}{|c|}{ Fatores relacionados } & $\mathbf{N}^{\mathbf{0}}$ & $\%$ \\
\hline Distúrbio no padrão & \multirow{2}{*}{24} & - Dificuldade para dormir & 02 & 95,8 \\
do sono & & - Sono interrompido & 20 & $\mathbf{8 3 , 3}$ \\
& & - Acorda cansado & 18 & 75,0 \\
& & - Uso de medicação para dormir & 01 & 4,1 \\
& & - Sonolento & 01 & 4,1 \\
\hline
\end{tabular}

O diagnóstico de distúrbio no padrão de sono é definido como interrupção no período do sono causando desconforto ou interferindo no estilo de vida desejado (FARIAS 1990). Observa-se no QUADRO 7 que dos 24 pacientes com esse diagnóstico $23(95,8 \%)$ se queixavam de dificuldade para dormir. Além disso, outras duas características que se destacaram foram: $20(83,3 \%)$ pacientes reclamaram de sono interrompido e $18(75 \%)$ referiram acordar cansados. Tem se destacado como característica definidora importante desse diagnostico a ausência de sensação de repouso ao acordar. Seis dos 24 pacientes não referiam esses dados, mas apesar disso, consideramos as outras características associadas suficientes para afirmar o diagnóstico para esses doentes. Em algumas situações essa resposta pareceu ser de extremo impacto para o próprio doente, que às 
vezes manifes tou que tudo o que necessitava durante a permanência na UTI era alguns períodos de sono repousante. OQUADRO 8 mostra os fatores relacionados desse diagnóstico elucidando melhor essa resposta.

QUADRO 8 - Freqüência dos fatores relacionados do diagnóstico de distúrbio no padrão do sono em pacientes internados em UTI. São Paulo, 1995.

\begin{tabular}{l|c|l|c|c}
\hline \multicolumn{1}{c|}{ Diagnostico } & $\mathbf{N}^{\mathbf{0}}$ & \multicolumn{1}{|c|}{ Fatores rclacionados } & $\mathbf{N}^{\mathbf{0}}$ & $\%$ \\
\hline Distúrbio no padrão & 24 & - Mudança ambiental & 24 & 100 \\
do sono & & - Dor & 07 & 29,1 \\
& & - Ansiedade & 04 & 16,6 \\
\hline
\end{tabular}

No QUADRO 8 observa-se que para todos os pacientes com distúrbio no padrão do sono, a mudança ambiental foi o fator relacionado que algumas vezes estava associada a outros (dor - 20,1\%; ansiedade - 16,6\%). Esses doentes confirmaram também a idéia de a unidade de terapia intensiva ser um ambiente estressante, pouco próprio ao sono repousante. Mesmo nos casos em que o doente tinha, aparentemente, condições para dormir a movimentação, ruídos e iluminação impediam que ele o fizesse caracterizando condições de superestimulação sensorial. Os efeitos da privação do sono associada a essa superestimulação certamente trazem efeitos deletérios para o paciente, mas o controle ambiental parece ser um dos desafios mais dificeis de enfrentar quanto a qualidade da assistência na UTI.

QUADRO 9 - Freqüência das características que fundamentaram o diagnóstico de dor em pacientes internados em UTI. São Paulo, 1995.

\begin{tabular}{l|c|l|c|c}
\hline \multicolumn{1}{c|}{ Diagnóstico } & $\mathbf{N}^{\mathbf{0}}$ & \multicolumn{1}{|c|}{ Características } & $\mathbf{N}^{\mathbf{0}}$ & $\%$ \\
\hline Dor & 20 & $\begin{array}{l}\text { - Refere dor } \\
\text { - Comportamento protetor }\end{array}$ & 20 & 05 \\
\hline
\end{tabular}

Observa-se no QUADRO 9 que o diagnóstico de dor foi formulado para 20 pacientes. Esse diagnóstico é definido como o estado em que o indivíduo experimenta e relata a presença de certo desconforto ou uma sensação desconfortável (FARIAS 1990). A dor foi caracterizada em $100 \%$ pela referência verbal do paciente. Em 25\% dos pacientes com dor também foi identificado o comportamento protetor como característica desse diagnóstico. A referência verbal foi observada em 11 pacientes submetidos a cirurgias.

QUADRO 10 - Freqüência dos fatores relacionados do diagnóstico de dor de pacientes internados em UTI. São Paulo, 1995.

\begin{tabular}{l|c|l|c|c}
\hline \multicolumn{1}{c|}{ Diagnóstico } & $\mathbf{N}^{\circ}$ & \multicolumn{1}{|c|}{ Fatores Relacionados } & $\mathbf{N}^{\circ}$ & $\%$ \\
\hline Dor & 20 & $\begin{array}{l}\text { - Injúria fisica } \\
\text { - Imobilidade no leito }\end{array}$ & 17 & 85 \\
& & & 03 & 15 \\
\hline
\end{tabular}


Dos fatores relacionados. em $17(85 \%)$ pacientes foi indicada a injúria física (cirurgias, trauma por entubação ferindo mucosa oral e isquemia miocárdica). A imobilidade do paciente no leito também mostrou contribuir para a ocorrência da dor. A queixa neste sentido foi de dor lombar necessitando a atuação da enfermagem na mudança de decúbito para seu alívio.

O QUAIJRO 11 a seguir mostra a distribuição dos diagnósticos formulados para a população segundo os Padrões Funcinais de Saúde propostos por GORDON (1994).

QUADRO 11 - Frequência dos diagnósticos de enfermagem formulados para doentes internados em UTI agrupados em padrões funcionais de saúde. ( $\mathrm{N}=256)$ São Paulo, 1995.

\begin{tabular}{|c|c|c|c|}
\hline Padrões & Diagnósticos & $\mathbf{N}^{\mathbf{0}}$ & $\%$ \\
\hline $\begin{array}{l}\text { Nutricional- } \\
\text { Metabólico }\end{array}$ & $\begin{array}{l}\text { - Alto risco para infecção } \\
\text { - Integridade da pele prejudicada } \\
\text { - Alteração na mucosa oral } \\
\text { - Alto risco para déficit de volume de líquido } \\
\text { - Alteração na nutriçāo para mais que o corpo necessita } \\
\text { - Excesso de volume de líquido } \\
\text { - Deglutição prejudicada } \\
\text { - Hiperternia } \\
\text { - Alteração na nutrição para menos que o corpo necessita } \\
\text { - Alteração na perfusão tecidual }\end{array}$ & $\begin{array}{l}31 \\
31 \\
07 \\
06 \\
04 \\
04 \\
03 \\
02 \\
02 \\
01\end{array}$ & 35,5 \\
\hline Atividade-Exercício & $\begin{array}{l}\text { - Mobilidade física prejudicada } \\
\text { - Intolerância a atividade } \\
\text { - Déficit de auto-cuidado } \\
\text { - Padrão respiratório alterado } \\
\text { - Elininaçâo traqueo-brônquica inelicaz }\end{array}$ & $\begin{array}{l}26 \\
14 \\
14 \\
03 \\
01\end{array}$ & 22,6 \\
\hline $\begin{array}{l}\text { Cognitivo- } \\
\text { Perceptivo }\end{array}$ & $\begin{array}{l}\text { - Dor } \\
\text { - Alteração senso perceptiva (audição) } \\
\text { - Alteração senso perceptiva (visual) } \\
\text { - Comunicação verbal prejudicada }\end{array}$ & $\begin{array}{l}20 \\
04 \\
03 \\
02\end{array}$ & 11,3 \\
\hline Sono e Repouso & - Distúrbios no padrāo do sono & 24 & 9.4 \\
\hline $\begin{array}{l}\text { Percepção e } \\
\text { Controle da Saúde }\end{array}$ & $\begin{array}{l}\text { - Déficit de conhecimento } \\
\text { - Alto risco para aspiração } \\
\text { - Potencial para trauma } \\
\text { - Alteração na manutenção da saúde }\end{array}$ & $\begin{array}{l}13 \\
06 \\
02 \\
01\end{array}$ & 8,6 \\
\hline Eliminações & $\begin{array}{l}\text { - Obstipação intestinal c constipação } \\
\text { - Diarréia } \\
\text { - Alteração da climinaçã̃o urinária }\end{array}$ & $\begin{array}{l}11 \\
05 \\
01\end{array}$ & 6.6 \\
\hline $\begin{array}{l}\text { Auto-Percepção e } \\
\text { Auto-Conceito }\end{array}$ & $\begin{array}{l}\text { - Ansiedade } \\
\text { - Medo } \\
\text { - Impotência }\end{array}$ & $\begin{array}{l}09 \\
03 \\
02\end{array}$ & 5,6 \\
\hline $\begin{array}{l}\text { Sexual } \\
\text { Reprodutivo }\end{array}$ & - Alterações no padrão de sexualidade & 01 & 0.4 \\
\hline
\end{tabular}


A freqüência dos diagnósticos encontrados foi maior para o padrão nutricional-metab6lico, possivelmente devido sua relação com fatores biológicos, ambientais e terapêuticos a que estão submetidos os pacientes críticos, sendo seguido do padrão de atividade-exercício que também apresenta uma expressão considerável, visto que, geralmente os pacientes em Unidades de Terapia Intensiva encontram-se limitados em suas atividades físicas. As demais frequências de diagnósticos por padrão foram gradativamente diminuindo, exceto em relação as do padrão sexual-reprodutivo. Talvez esse padrão não tenha sido adequadamente explorado, possivelmente devido a: interferência do meio ambiente, falta de vínculo entre pesquisador e paciente, ou ainda, por não se constituir naquele momento um problema relevante para o paciente. Destacase também que não houve a identificação de diagnosticos dos padrões: de crença e valor, tolerância e resposta ao estresse e de desempenho de papel e relacionamento.

\section{CONCLUSÕES}

Com a realização da pesquisa com 32 pacientes internados em Unidade de Terapia Intensiva, formulamos os diagnósticos de enfermagem e identificamos os padrões funcionais de saúde alterados com as seguintes conclusões:

Os diagnósticos de enfermagem formulados para mais de $50 \%$ dos pacientes foram: alto risco para infecção, integridade da pele prejudicada, mobilidade física prejudicada, distúrbio no padrão do sono e dor.

Os diagnósticos de enfermagem presentes entre $40,6 \%$ a $34,3 \%$ dos pacientes foram: déficit de conhecimento (da doença), intolerância a atividade, déficit para autocuidado (banho, vestir, eliminações), obstipação intestinal (constipação).

Os diagnósticos de enfermagem formulados para menos de $30 \%$ dos pacientes foram: ansiedade, alteração na mucosa oral, alto risco para aspiração, alto risco para déficit de volu me de líquido, diarréia, alteração na nutrição para maior que o corpo necessita, alteração senso perceptiva (audição) excesso de volume de líquido, medo, deglutição prejudicada, padrão respiratório ineficaz, alteração senso percep tiva (visual), comunicação verbal prejudicada, impotência, potencial para trauma, hipertermia, alteração na nutrição para menos que o corpo necessita, alteração no padrão da sexualidade, eliminações traqueo bronquica ineficaz, alteração na manutenção da saúde, alteração na perfusão tecidual, alteração da eliminação urinária.

Os padrões funcionais de saúde alterados foram: padrão nutricionalmetab́blico $(35,4 \%)$, de atividade-exercício $(22,6 \%)$, cognitivo-perceptivo $(11,3 \%)$, sono e repouso $(9,4 \%)$, percepção e controle da saúde $(8,6 \%)$, eliminações $(6,6 \%)$, auto-percepşão e auto-coneito $(5,6 \%)$, e padrão sexual-reprodutivo $(0,4 \%)$. 


\section{CONSIDERAÇÕES FINAIS}

O desenvolvimento desta pesquisa favoreceu a identificação das necessidades de cuidados de doentes internados em unidades de terapia intensiva e proporcionou oportunidade para o desenvolvimento da habilidade diagnóstica.

Neste processo de investigação a análise das entrevistas e dos dados do exame físico levou ao aprofundamento da especificidade do doente crítico com base nos diagnóstico de enfermagem da North American Nursing Diagnosis Association (NANDA).

No presente estudo, cuja população limitou-se a pacientes conscientes, obtivemos a média de 8 diagnósticos por paciente. Acreditamos que essa média poderia ser ainda maior, se englobássemos todos os pacientes encontrados em Unidades de Terapia Intensiva. Os resultados indicam a necessidade da replicação desse estudo incluindo pacientes com alteração de nível de consciência, o que 6 frequente em unidades de terapia intensiva, a fim de melhor compor o perfil de necessidades desses doentes. () controle de outras variáveis e posterior comparação, como por exemplo, o tempo de internação em UTI poderia fornecer outras informações a respeito da evolução e gravidade dos pacientes de UTI. A utilização dos dados de apenas uma entrevista com cada doente é também uma das limitações deste estudo.

PASINI,D. et al Nursing diagnoses of patients of critical care unit. Rev. Esc. Enf. USP, v. 30 , n.3, p.501-18, dec. 1996.

The aim of this study was to identify the nursing care needs of patients of critical care unit. The nursing diagnoses of 32 patients was formulated. The data was collected by interview and physical examination and the Functional Health Patterns was the framework to collect the data and to identify the predominant dysfunctional health areas.

UNITERMS: Nursing diagnoses. Critical care unit. 


\section{ANEXO I}

\section{Parte I}

Dados de Identificaça:

Instituição:

Diagnóstico(s) Médico(s):

Nome: Idade:

Sexo:

Estado Civil: Escolaridade:

Ocupação: Dias de Internação - na U.T.I.:

Total:

\section{Parte II}

\section{Entrevista com o Paciente:}

1- Padrão de Percepção e controle da Saúde:

1.1- Qual o motivo que o trouxe para U.T.I.?

1.2- Já esteve internado em alguma U.T.I. anteriormente?

( ) Sim

( ) Năo

1.3- Como foi sua internação anterior na U.T.I.?

2 - Padrão Nutricional Metabólico:

2.1- Como está sua aceitação alimentar?

2.2- Como está sua ingestão de líquidos?

3- Padrão de eliminação:

3.1- Como está o funcionamento de seu intestino?

3.2- Como está o funcionamento de sua bexiga?

4- Padrão de atividade-exercício:

4.1- Tem alguma dificuldade de movimentar-se no leito?
( ) Não
( ) Sim
Qual(is)?

4.2- O(a) Sr.(a) faz algum tipo de exercicio ativo no leito?
( ) Não
( ) Sim
Por quê?

5- Padrão de sono e repouso:

5.1- $\mathrm{O}$ (a) $\mathrm{Sr}$ (a) tem conseguido dormir?
( ) Não
( ) Sim
Qual(is)?

5.2- Existem fatores que tem interferido no seu sono?
( ) Não
( ) Sim
Qual(is)?

5.3- Quando dorme, acorda descansado(a)?
( ) Não
( ) Sim
Por quê?

6- Padrão Cognitivo-Perceptivo:

6.1- Tem alguma dificuldade para ver?

6.2- Tem alguma dificuldade para ouvir?

6.3- O que o(a) incomoda neste ambiente de U.T.I.?

7- Padrão de auto percepção e auto-conceito:

7.1- Como o(a) $\mathbf{S r}(a)$ se descreveria ?

8- Padrão de desempenho de papel e relacionamento:

8.1- A doença interferiu no seu relacionamento familiar? 
9- Padrão Sexual-Reprodutivo:

9.1- $\mathrm{O}$ (a) $\operatorname{Sr}$ (a) tem alguma preocupação quanto a sua atividade sexual?

10- Padrão de Tolerância e Resposta ao Estresse:

10.1- O que é estar numa U.T.I. para o(a) $\mathbf{S r}(a)$ ?

10.2- Como o(a) $\operatorname{Sr}(a)$ procura trabalhar essa situaçåo?

11- Padrăo de Crença e Valor:

11.1- Existem coisas espirituais importantes para o $\operatorname{Sr}(a)$ ?

11.2- Elas o ajudam em seus momentos dificeis?

12- Outros:

12.1- Há alguma coisa que não tenhamos falado que o $\operatorname{Sr}(a)$ gostaria de perguntar?

$$
\text { () Năo ( ) Sim Por quê? }
$$

\section{Parte III}

1. Dados Físicos e Observações Durante a Entrevista:

Temperatura Axilar:

Pulso: Frequência:

Ritmo:

Respiração:

Pressão Arterial:

Peso:

Altura:

Peso Ideal:

- Cabeça:

Cabelos:

Orelhas:

Olhos:

Nariz:

Boca:

- Membros Superiores e Inferiores:

Movimentação

Musculatura:

Condigres da pele e anexos:

- Tronco:

Pele:

Ausculta Cardíaca

Ausculta Pulmonar:

Ausculta Abdominal:

Outras Alterações:

- Abdome:

- Alimentação:

( ) Via Oral ( ) Via Enteral ( ) Jejunostomia ( ) Gastrostomia （ ) Parenteral

- Genitais:

- Impresgões do Examinador:

- Equipamentos Terapêuticos/Diagnósticos: 


\section{REFERÊNCIAS BIBLIOGRÁFICAS}

BUB, M. B. C. et al. Sistematização da assistência de enfermagem em terapia intensiva e a classificaçăo diagnóstica da NANDA. In: SIMPÓSIO INTERNACIONAL SOBRE DIAGNÓSTICO DE ENFERMAGEM. 1“. São Paulo. 1995. Programa. São Paulo, EeUSP, 1995. p.52.

CREASON, N. S. et al. Validating the nursing diagnosis of impaired physical mobility. Nurs.Clin.NorthAm. v.20, n.4, p.669-83, 1985

CRUZ, D. A. L. M da. Diagnósticos de enfermagem e padrões funcionais de saúde alterados de pacientes internados por cardiopatia chagásica crônica. Sáo Paulo, 1989. 92p. Dissertação (mestrado) - Escola de Enfermagem, Universidade de São Paulo.

CRUZ, D. A. L. M. da. Diagnóstico de enfermagem: aspectos históricos e definição. Rev.Paul.Enf., v. 13, n.1/3. p. 3-7. 1994.

CRUZ, I.C.F. da. Diagnóstico de enfermagem: estratégia para sua formulação e validação. São Paulo, 1993. 157p. Tese (doutorado). Escola de Enfermagem. Universidade de São Paulo.

FARIAS, F, et al. Diagnóstico de enfermagem: uma abordagem conceitual e prática. Joăo Pessoa, Santa Marta, 1990.

GALVÃO. S. R. dos S: CRUZ D. A. L. M. da. Estudo dos fatores de risco para o diagnóstico de alto risco para infecção. In: CONGRESSO BRASILEIRO DE ENFERMAGEM EM CENTRO CIRÚRGICO. 2. São Paulo. 1995. Anais. São Paulo. Associação Brasileira de Enfermeiros de Centro Cirúrgico, São Paulo, 1995. p.155-67.

GEBBIE. K. M.; LAVIN. M. A. National Conference on Nursing Diagnosis Classification, 1: Proceedings. Saint Louis, Mosby. 1975.

GREENLEE, K. K. The effects of implementation of an operational definition and guidelines for the formulation of nursing diagnosis in critical care setting. In: CARROLL-JOHNSON, R. M. (ed) National Conference on Classification of Nursing Diagnosis 9: Proceedings. Philadelphia. Lippincott, 1991, p.260-1.

GORDON. M. et al. Nursing diagnosis: looking at its use in the clinical area. Am. J. Nurs. v.80, n.4. p.672-74, 1980 .

GORDON, M. High-risk nursing diagnoses in critical care. In: CARROL-JOHNSON,R. M., PAQUETTE, M.(ed.) Classification of Nursing Diagnosis: proceedings of the tenth Conference of the North American Nursing Diagnosis Association. Philadelphia, Lippincott, 1994. p.250-4.

GORDON, M. Nursing diagnosis: process and application. 3ed. St. Louis. Mosby. 1994

GORDON, M. Nursing diagnosis: process and application. 2ed. New York. McGraw-Hill. 1987.

GORDON, M. Nursing diagnosis: process and application. St. Louis. McGraw-Hill, 1982. 
HOSKINS, L. M. et al. Nursing diagnosis in the chronically ill: methodology for clinical validation. ANS, v.8, n.3, p.80-9,1986.

MARIA, V. L. R. Preparo de enfermeiros para utilização de diagnósticos de enfermagem: relato de experiência. São Paulo, 1990. 130p. Dissertação (mestrado). Escola de Enfermagem, Universidade de São Paulo.

McDONALD. B. R. Validation of three respiratory nursing diagnoses. Nurs. Clin. North Am., v.20, n.4, p.697-710. 1985 .

MORRIS, C. A. Self-concept as altered by the diagnosis of cancer. Nurs. Clin. North Am. v.20, n.4, p.611-30, 1985 .

MIILS, W. C. Nursing diagnosis: the importance of a definition. Nurs. Diag. v.2. n.1. p.3-8. 1991.

MUNDIGER, M. O. Nursing diagnoses for cancer patients. Cancer Nurs., v.1. n.3. p.221-6. 1978.

MUNNS. D. C. Avalidation of the defining characteristics of the nursing diagnosis $>$ "potential for violence" Nurs. Clin. North Ain., v.20, n.4, p.711-22. 1985.

NORTH AMERICAN NURSING DIAGNOSIS ASSOCIATION. Nursing diagnosis: definitions \& classification 1995-1996. Philadelphia. NANDA. 1994.

PIMENTA. C. A. de M.: CRUZ. D. A. L. M. da. Câncer e dor: alteraçōes nos padrões de respostas humanas. ACTA Paul.Enf. v.17, n.1, p. 27-34,1994.

SHOEMAKER, J. K. Characteristics of a nursing diafnosis. Occup. Health Nurs., v. 33, n.8. p.387-9. 1985.

WIESEKE. A et al. A content validation study of five nursing diagnoses by critical care nurses. Heart Lung. v.23. n.4. p.345-51. 1994.

VINCENT. K. G. The validation of a nursing diagnosis: a nurse consensus survey. Nurs. Clin. North Aln. v. 20, n.4, p.631-40, 1985.

VOITH. A. M.: SMITH. D. A. Validation of the nursing diagnosis of urinary retention. Nurs. Clin North Am., v.20. n.4. p.723-30. 1985. 